\title{
PROBLEMA DAS LUZES APAGADAS
}

\section{Light Off Problem}

\section{Natalia Rodrigues da Silva ${ }^{1}$; Hudson Alves Martins ${ }^{2}$; Fernando Pereira de Souza ${ }^{3}$}

\author{
${ }^{1}$ Universidade Federal de Mato Grosso do Sul \\ Departamento de Ciências Exatas \\ ntl rodrigues@outlook.com
}

\author{
${ }^{2}$ InstitutoFederal de Mato Grosso do Sul \\ Professor EBTT \\ hudson.martins@ifms.edu.br
}

\author{
${ }^{3}$ Universidade Federal de Mato Grosso do Sul \\ Departamento de Ciências Exatas \\ fermatmel@gmail.com
}

RESUMO - A Álgebra Linear apresenta um papel muito importante nas áreas de exatas, e através dela podemos mostrar sua utilidade na modelagem de um problema que envolve um simples jogo de apagar e acender luzes, depois de modelado o problema, vamos resolve-lo implementando um algoritmo de eliminação de Gauss no Scilab (o Scilab é um software livre e de código aberto, voltado para computação numérica semelhante ao Matlab). O jogo, Lights Out, é um famoso jogo da década de 90, o qual consiste de 25 teclas iluminadas e arranjadas na forma de uma matriz 5x5, onde se tem um estado inicial e devemos apagar todas as teclas apertando uma sequência correta de teclas, esta sequência será fornecida pelo programa implementado no Scilab, ou seja teremos uma solução imediata para apagarmos todas as luzes do jogo. A modelagem utiliza os conceitos de Álgebra Linear: matrizes, determinantes e sistemas lineares, juntamente com o conceito de conjunto dos resto da divisão por $2\left(\mathbb{Z}_{2}\right)$.

Palavras-chave: jogo luzes apagadas; apagar e acender luzes; problemas das luzes.

ABSTRACT - Linear Algebra presents a very important role in the areas of accuracy, and through it we can show its utility in modeling a problem that involves a simple game of erasing and lighting lights, after modeling the problem, we will solve it by implementing an algorithm of elimination of Gauss in Scilab (Scilab is free and open source software, focused on numerical computation similar to Matlab). The game, Lights Out, is a famous game of the 90 's, which consists of 25 keys illuminated and arranged in the form of a $5 \times 5$ matrix, where it has an initial state and we must delete all keys by pressing a correct sequence of keys, this sequence will be provided by the program implemented in Scilab, ie we will have an immediate solution to erase all lights in the game. The modeling uses Linear Algebra concepts: matrices, determinants and linear systems, together with the concept of the set of the rest of the division by $2\left(\mathbb{Z}_{2}\right)$.

Keywords: game lights off; turn lights on and off; light problems.
Revisado em: 21/08/2018

Aprovado em: 29/08/2018 


\section{INTRODUÇÃO}

O jogo Lights Out consiste em uma grade de $5 \times 5$ de luzes. Ao iniciarmos o jogo, uma configuração é nos dada com um número aleatório ou padrão de luzes. Pressionar qualquer uma das luzes irá alterná-lo e as luzes que estão diretamente à direita, à esquerda, acima e abaixo tem seu estado alterado. Este jogo tem como objetivo desligar todas as luzes, de preferência no menor número possível de pressionamentos de botão. Para isso utilizamos matrizes, sistemas lineares e $\mathbb{Z}_{2}$ e o software Scilab. Estas ferramentas nos auxiliam para a obtenção de uma solução rápida para o jogo, assim não haverá a necessidade de termos que apertar as teclas de modo que apagaremos todas $s$ luzes nas "tentativas e erros", ou seja, tentaremos um método, e observaremos se ele funciona, e se não funcionar tentaremos um novo método, apertaremos uma nova sequência de teclas. No entanto, este não será nosso caso, pois teremos a Álgebra Linear para nos auxiliar e poupar nosso tempo. No jogo teremos luzes acesas e apagas, e desta forma atribuiremos 1 para as luzes acesas e 0 para as luzes apagadas, com a nossa configuração inicial do jogo, teremos um vetor $b \in \mathbb{Z}_{2}$ que será a sequências de 0 e 1 de acordo com a disposição das luzes, acesas ou apagadas, e $\mathrm{x}$ será o estado obtido quando apertamos um determinada sequência de teclas, além disso teremos um sistema $T$ que é o nosso estado obtido quando temos todas as nossas luzes apagadas e apertamos todas as teclas uma vez, logo teremos um sistema linear na forma matricial $A x=T$ com $T \in \mathbb{Z}_{2}$ e a $A_{25 \times 25}$. Para resolver o problema das luzes apagadas, temos que achar a solução para $A x=T$, e neste caso utilizaremos o software Scilab onde iremos implementar um programa que consiste em receber o estado inicial do jogo, verificar se tem solução e por fim se tiver, nos fornecerá a solução, ou seja, a sequência de teclas que serão apertadas.

\section{METODOLOGIA}

No desenvolvimento deste trabalho foram realizados estudos teóricos sobre a resolução de matrizes, e sistemas lineares, juntamente com o conceito de conjunto dos restos da divisão por $2\left(\mathbb{Z}_{2}\right)$. Foi trabalhada a parte de escalonamento no conjunto $\mathbb{Z}_{2}$, além de analisarmos alguns exemplos de configurações do jogo e como se dispunham na forma matricial e em um sistema linear. Para uma melhor compreensão do assunto abordado, testamos todo o conteúdo na prática ao jogarmos o Light Out, além disso, foram feitas apresentações de seminários com a presença do orientador.

\section{RESULTADOS}

\subsection{Modelando o Problema das Luzes Apagadas}

Começando o jogo com uma determinada configuração, por exemplo:

Figura 1. configuração inicial

\begin{tabular}{|l|l|l|l|l|}
\hline 1 & 2 & 3 & 4 & 5 \\
\hline 6 & 7 & 8 & 9 & 10 \\
\hline 11 & 12 & 13 & 14 & 15 \\
\hline 16 & 17 & 18 & 19 & 20 \\
\hline 21 & 22 & 23 & 24 & 25 \\
\hline
\end{tabular}

Neste jogo, não há a necessidade de apertarmos as teclas mais que uma vez, pois apertando uma quantidade par de vezes, ela retorna ao seu estado inicial e apertando uma quantidade ímpar de vezes, ela permanece no mesmo estado quando apertamos pela primeira vez. Seja "1" indicando o estado aceso da tecla e " 0 " o estado apagado da tecla, a configuração inicial do jogo representado acima pode ser indicado pelo vetor $b \in\left(\mathbb{Z}_{2}\right)^{25}$ 
$$
b=\left(\begin{array}{l}
0 \\
0 \\
1 \\
0 \\
0 \\
1 \\
1 \\
0 \\
1 \\
1 \\
0 \\
0 \\
1 \\
0 \\
0 \\
0 \\
1 \\
0 \\
1 \\
0 \\
1 \\
0 \\
0 \\
0 \\
1
\end{array}\right)
$$

Se a condição do nosso estado inicial é todas as luzes apagadas e formos apertando as teclas aleatoriamente, chegaremos num estado que tem solução, pois se apertamos as mesmas teclas que já foram apertadas, retornaremos ao nosso estado inicial, com todas as luzes apagadas. Este estado é obtido quando apertamos uma determinada sequência de teclas, e que chamaremos de:

$$
x=\left(x_{1}, x_{2}, x_{3}, \ldots, x_{25}\right)^{t}
$$

Exemplo:

Figura 2. Estado Inicial

\begin{tabular}{|c|c|c|c|c|}
\hline 1 & 2 & 3 & 4 & 5 \\
\hline 6 & 7 & 8 & 9 & 10 \\
\hline 11 & 12 & 13 & 14 & 15 \\
\hline 16 & 17 & 18 & 19 & 20 \\
\hline 21 & 22 & 23 & 24 & 25 \\
\hline
\end{tabular}

$$
x=\left(\begin{array}{l}
1 \\
0 \\
0 \\
0 \\
0 \\
1 \\
0 \\
0 \\
1 \\
0 \\
0 \\
0 \\
0 \\
0 \\
0 \\
0 \\
1 \\
0 \\
0 \\
0 \\
0 \\
0 \\
1 \\
0 \\
0
\end{array}\right)
$$

Figura 3. Sequência $x$ apertada

\begin{tabular}{|l|l|l|l|l|}
\hline 1 & 2 & 3 & 4 & 5 \\
\hline 6 & 7 & 8 & 9 & 10 \\
\hline 11 & 12 & 13 & 14 & 15 \\
\hline 16 & 17 & 18 & 19 & 20 \\
\hline 21 & 22 & 23 & 24 & 25 \\
\hline
\end{tabular}

Seja $T=\left(\begin{array}{lllll}t_{1} & t_{2} & t_{3}, \ldots, & t_{25}\end{array}\right)^{t}$ o estado obtido quando temos todas as luzes apagadas e apertamos todas as teclas uma vez:

$$
\begin{array}{rlrrrr}
t_{1} & = & & x_{1}+x_{2}+x_{6} \\
t_{2} & = & & x_{1}+x_{2}+x_{3}+x_{6} \\
t_{3}= & & x_{2}+x_{3}+x_{4}+x_{8} \\
& \vdots & & & \vdots \\
t_{12} & = & x_{7}+x_{11}+x_{12}+x_{13}+x_{17} \\
& \vdots & & \vdots \\
t_{24} & = & & x_{19}+x_{23}+x_{24}+x_{25} \\
t_{25}= & & x_{20}+x_{24}+x_{25}
\end{array}
$$

O sistema Linear acima pode ser escrito na forma matricial $A x=T \operatorname{com} T \in\left(\mathbb{Z}_{2}\right)^{25}$ e $A_{25 \times 25}$, com: 


$$
A=\left[\begin{array}{lllll}
B & I & 0 & 0 & 0 \\
I & B & I & 0 & 0 \\
0 & I & B & I & 0 \\
0 & 0 & I & B & I \\
0 & 0 & 0 & I & B
\end{array}\right]
$$

onde

$$
\begin{aligned}
B & =\left[\begin{array}{lllll}
1 & 1 & 0 & 0 & 0 \\
1 & 1 & 1 & 0 & 0 \\
0 & 1 & 1 & 1 & 0 \\
0 & 0 & 1 & 1 & 1 \\
0 & 0 & 0 & 1 & 1
\end{array}\right] \\
\text { e } I & =\left[\begin{array}{lllll}
1 & 0 & 0 & 0 & 0 \\
0 & 1 & 0 & 0 & 0 \\
0 & 0 & 1 & 0 & 0 \\
0 & 0 & 0 & 1 & 0 \\
0 & 0 & 0 & 0 & 1
\end{array}\right]
\end{aligned}
$$

Matriz A:

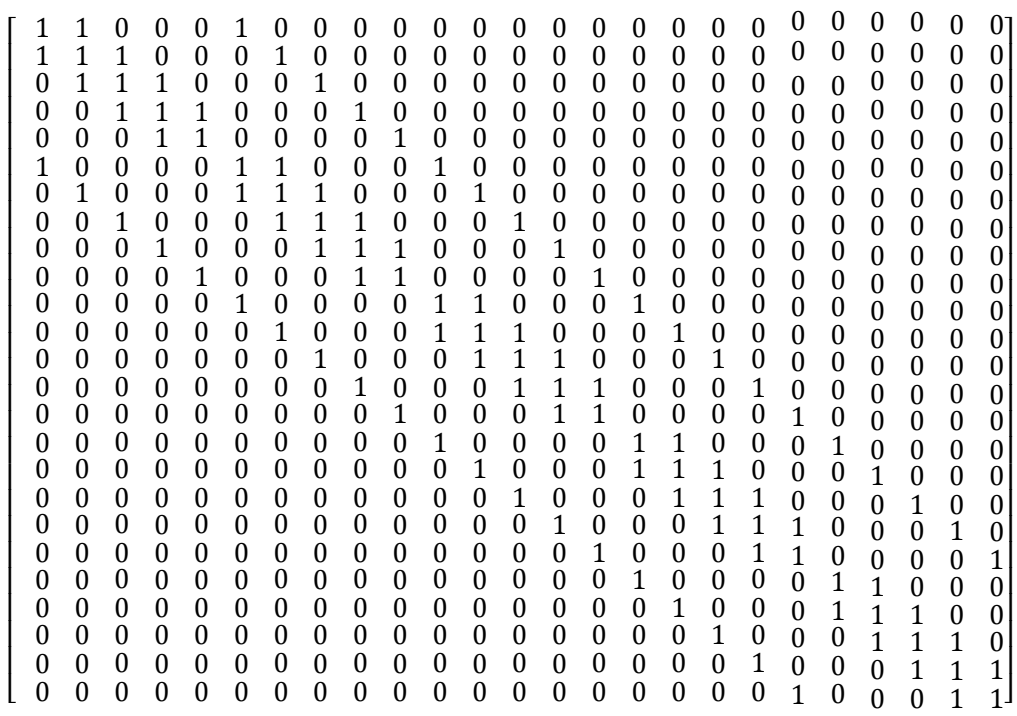

Um método deve ser encontrado para que haja solução de um estado $b$, ou seja, uma estratégia para que:

$$
A x+b=0
$$

Como estamos trabalhando no conjunto $\mathbb{Z}_{2}$, logo $b \in\left(\mathbb{Z}_{2}\right)^{25}$, então se somarmos $b$ em ambos os lados temos que $A x=b$, e assim resolveremos este sistema. No entanto, um estado $b$ pode ser resolvido se e somente, se $b$ pertencer ao espaço coluna de $A(b \in \operatorname{col}(A))$. Neste caso, $A$ é simétrica, então seu espaço coluna $(\operatorname{col}(A))$ é igual ao espaço linha $(\operatorname{row}(A))$. Entretanto, o espaço linha de $A$ é o complemento ortogonal do espaço nulo $A(\operatorname{Ker}(A))$. Desta forma, a matriz $E$ é a forma escalonada de $A$, portanto, teremos:

Matriz E:

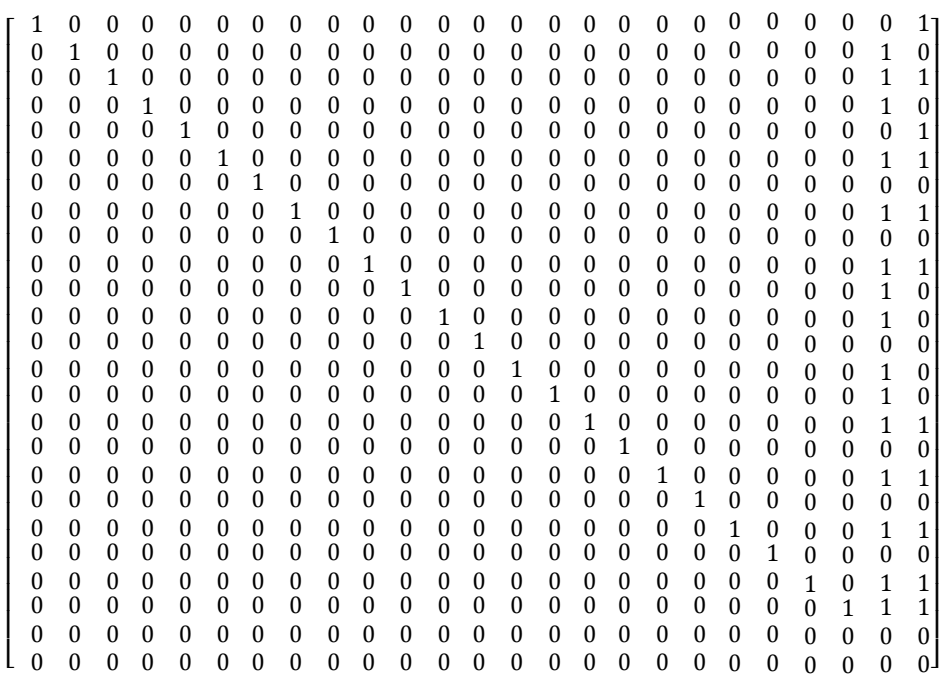

Assim, $\operatorname{Ker}(A)=\operatorname{Ker}(E)$ e o Posto $(A)=$ 23, logo a $\operatorname{dim}(\operatorname{Ker}(A))=2$. As variáveis livres estão nas duas últimas colunas da matriz $E$. Ou seja, $\operatorname{col}(A)=\operatorname{row}(A)$ é gerado pelas 23 primeiras linhas.

$0 \operatorname{col}(A)$ é formado pelos vetores ortogonais aos vetores da base do espaço nulo de A e como as duas últimas colunas são dadas por:

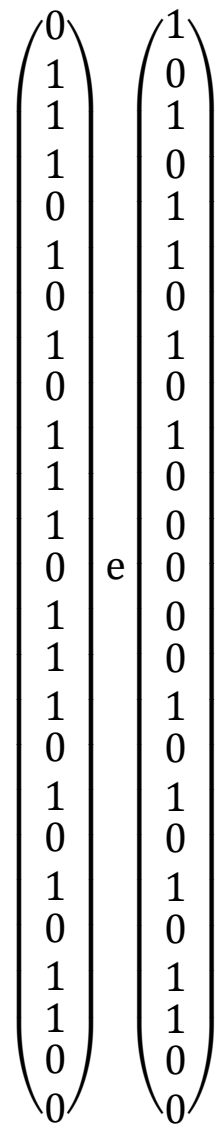

Temos que a base do espaço nulo de $A$ é dada pelos vetores: 
$\underset{n_{1}}{\longrightarrow}\left(\begin{array}{l}0 \\ 1 \\ 1 \\ 1 \\ 0 \\ 1 \\ 0 \\ 1 \\ 0 \\ 1 \\ 1 \\ 1 \\ 1 \\ 1 \\ 1 \\ 0 \\ 1 \\ 0 \\ 1 \\ 0 \\ 1 \\ 1 \\ 0 \\ 0\end{array}\right) \mathrm{e} \underset{n_{2}=}{\longrightarrow}\left(\begin{array}{l}1 \\ 0 \\ 1 \\ 0 \\ 1 \\ 1 \\ 0 \\ 0 \\ 0 \\ 1 \\ 0 \\ 1 \\ 0 \\ 1 \\ 0 \\ 1 \\ 0 \\ 1 \\ 1 \\ 0 \\ 0\end{array}\right)$

O sistema $A x=b$ só terá solução se, $\mathrm{e}$ somentese, se $b$ é ortogonal a $\overrightarrow{n_{1}}$ e $\overrightarrow{n_{2}}$. Se $b$ é ortogonal a $\overrightarrow{n_{1}}$ e $\overrightarrow{n_{2}}$, temos que $b \in \operatorname{col}(A)$. Portanto, $A x=b$ tem solução.

\subsection{A solução}

Para obtermos a solução do nosso sistema linear $A x=b$, primeiramente utilizamos o método de eliminação de Gauss, que consiste em aplicar sucessivas operações elementares à matriz aumentada do sistema, até que ela fique na forma escalonada reduzida, equivalente a matriz original. Este método nos permite verificar se tal sistema tem solução ou não.

Se o sistema tiver solução, utilizaremos o software Scilab que nos possibilita obter a solução imediata, assim para resolver o problema das luzes apagadas, implementamos o seguinte programa no Scilab:

1 clear; \%Limpando dados do Scilab

2 clc;

3 disp("Resolvedo o Problema de Apagar todas as Luzes ..");
4 disp("
ByHudson Martins

5 I=eye(5,5); \%Gerando a Matriz Identidade

$5 \times 5$

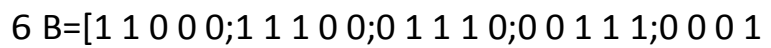

1]; \%Inserindo a Matriz $B$
7 o=zeros(5,5); \%Criando Matriz de zeros5x5;

$8 \mathrm{~A}=[\mathrm{B}$ I $\circ$ o o;। B I O o; O I B I o; O ० I B I; O ० ० । B];

$\%$ Criando a matriz $A$

9 c=zeros(1,25); \%Gerando o Estadoinicial 10 for $m=1: 25$;

$11 \mathrm{c}(\mathrm{m})=$ input("digite o estado inicial da casa")

12 disp("voce digitou a casa",m)

13 end

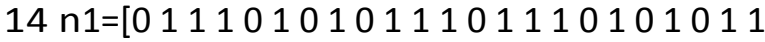

0 0];

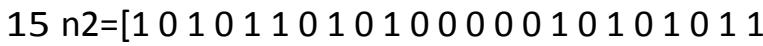

0 0];

16 e1=c*n1'; \%verificando se o estado inicial tem Resultado!!

17 e1=modulo(e1,2);\%Utilizando restos por 2

18 e2=c*n2';

19 e2=modulo(e2,2);

20 if e1==1 then

21 disp("Estado inicial sem Resultado!!")

22 elseif e2==1

23 disp("Estado Inicial sem Resultado!!")

24 else

$25 \mathrm{Ab}=\left[\mathrm{Ac} \mathrm{c}^{\prime}\right] ; \quad$ \%Resolvendo o Sistema $\mathrm{Ax}=\mathrm{B}$

26 for $\mathrm{j}=1: 24$;

27 for $k=j+1: 25$ \%pivoteamento

28 if $A b(j, j)<A b(k, j)$

29 Aux $=A b(j,:)$;

$30 \mathrm{Ab}(\mathrm{j},:)=\mathrm{Ab}(\mathrm{k},:)$;

$31 \mathrm{Ab}(\mathrm{k},:)=\mathrm{Aux}$;

32 end

33 end

34 for $\mathrm{i}=\mathrm{j}+1: 25$;\%Transformando a matriz

Aumentada em Uma Matriz TriangularSuperior

35 if $A b(i, j)==1$ then

$36 \mathrm{Ab}(\mathrm{i},:)=A b(\mathrm{i},:)+A b(\mathrm{j},:)$;

$37 \mathrm{Ab}(\mathrm{i},:)=$ modulo $(A b(\mathrm{i},:), 2)$;

38 end

39 end

40 end

41 for I=25: 1:2; \%Escrevendo Matriz na forma escadareduzida.

42 for $n=1: 1: 1$

43 if $A b(n, I)==1$ then

44 end

45 end

46 for I=25:-1:2; //Escrevendo Matriz na forma

escada reduzida.

48 for $n=1-1:-1: 1$

49 if $A b(n, I)==1$ then

"); $50 \mathrm{Ab}(\mathrm{n},:)=A b(\mathrm{n},:)+A b(\mathrm{l},:)$;

$51 \mathrm{Ab}(\mathrm{n}, \mathrm{:})=\underline{\text { modulo }}(\mathrm{Ab}(\mathrm{n}, \mathrm{:}), 2)$;

52 end

53 end

54 end 
$55 \mathrm{~s}=\mathrm{Ab}(:, 26)$;

56 disp("A Solução é :");

$\mathrm{so}=[\mathrm{s}(1) \mathrm{s}(2) \mathrm{s}(3) \mathrm{s}(4) \mathrm{s}(5) ; \mathrm{s}(6) \mathrm{s}(7) \mathrm{s}(8) \mathrm{s}(9) \mathrm{s}(10)$;

$s(11) s(12) s(13) s(14) s(15) ; s(16) s(17) s(17) s(19)$

s(20); s(21) s(22) s(23) s(24) s(25)] //Colocando a

Solução na forma $5 \times 5$

57 end

Tabela 1. Interpretação das linhas do Scilab

\begin{tabular}{|l|l|}
\hline Linhas & Descrição das linhas do programa \\
\hline 1 e 2 & Limpa as variáveis do Scilab \\
\hline 3 e 4 & Cabeçalho do programa \\
\hline 5 à 8 & Criando a Matriz $A$ \\
\hline 9 à 13 & Gerando o estado inicial \\
\hline 14 e & Gerando bases do espaço nulo \\
15 & \\
\hline 16 à & Analisando se o estado inicial tem \\
19 & solução \\
\hline 20 à & Para!! o programa se a condição inicial \\
23 & não for satisfeita \\
\hline 24 à & Resolve o sistema linear com \\
39 & pivoteamento \\
\hline 41 à & Transforma a matriz aumentada numa \\
48 & matriz na forma escada reduzida \\
\hline 49 e & Escreve o resultado \\
\hline 50 & \\
\hline 51 & Finaliza o programa \\
\hline
\end{tabular}

O programa funciona da seguinte forma, ele recebe o estado inicial do jogo, em seguida verifica se tem solução, e se o estado inicial tiver solução, logo ele nos fornece as teclas que devem ser apertadas para apagarmos todas as luzes, e assim atingiremos o nosso objetivo no jogo.

Exemplo: Seja o estado inicial do jogo

Figura 4. Configuração Inicial

\begin{tabular}{|c|c|c|c|c|}
\hline 1 & 2 & 3 & 4 & 5 \\
\hline 6 & 7 & 8 & 9 & 10 \\
\hline 11 & 12 & 13 & 14 & 15 \\
\hline 16 & 17 & 18 & 19 & 20 \\
\hline 21 & 22 & 23 & 24 & 25 \\
\hline
\end{tabular}

Utilizando o programa encontramos a seguinte solução:

$$
\left[\begin{array}{lllll}
1 & 1 & 1 & 0 & 0 \\
1 & 0 & 1 & 0 & 1 \\
0 & 0 & 0 & 1 & 1 \\
1 & 0 & 1 & 0 & 1 \\
1 & 1 & 1 & 0 & 0
\end{array}\right]
$$

Vejamos o que acontece quando seguimos essa sequencia:

\begin{tabular}{|c|c|c|c|c|}
\hline 1 & 2 & 3 & 4 & 5 \\
\hline 6 & 7 & 8 & 9 & 10 \\
\hline 11 & 12 & 13 & 14 & 15 \\
\hline 16 & 17 & 18 & 19 & 20 \\
\hline 21 & 22 & 23 & 24 & 25 \\
\hline
\end{tabular}

Tecla 1 apertada

\begin{tabular}{|c|c|c|c|c|}
\hline 1 & 2 & 3 & 4 & 5 \\
\hline 6 & 7 & 8 & 9 & 10 \\
\hline 11 & 12 & 13 & 14 & 15 \\
\hline 16 & 17 & 18 & 19 & 20 \\
\hline 21 & 22 & 23 & 24 & 25 \\
\hline
\end{tabular}

Tecla 2 apertada

\begin{tabular}{|c|c|c|c|c|}
\hline 1 & 2 & 3 & 4 & 5 \\
\hline 6 & 7 & 8 & 9 & 10 \\
\hline 11 & 12 & 13 & 14 & 15 \\
\hline 16 & 17 & 18 & 19 & 20 \\
\hline 21 & 22 & 23 & 24 & 25 \\
\hline
\end{tabular}

Tecla 3 apertada

\begin{tabular}{|c|c|c|c|c|}
\hline 1 & 2 & 3 & 4 & 5 \\
\hline 6 & 7 & 8 & 9 & 10 \\
\hline 11 & 12 & 13 & 14 & 15 \\
\hline 16 & 17 & 18 & 19 & 20 \\
\hline 21 & 22 & 23 & 24 & 25 \\
\hline
\end{tabular}

Tecla 6 apertada 


\begin{tabular}{|c|c|c|c|c|}
\hline 1 & 2 & 3 & 4 & 5 \\
\hline 6 & 7 & 8 & 9 & 10 \\
\hline 11 & 12 & 13 & 14 & 15 \\
\hline 16 & 17 & 18 & 19 & 20 \\
\hline 21 & 22 & 23 & 24 & 25 \\
\hline \multicolumn{4}{|c|}{ Tecla 8 apertada }
\end{tabular}

\begin{tabular}{|c|c|c|c|c|}
\hline 1 & 2 & 3 & 4 & 5 \\
\hline 6 & 7 & 8 & 9 & 10 \\
\hline 11 & 12 & 13 & 14 & 15 \\
\hline 16 & 17 & 18 & 19 & 20 \\
\hline 21 & 22 & 23 & 24 & 25 \\
\hline \multicolumn{5}{|c}{ Tecla 16 apertada } \\
\hline
\end{tabular}

\begin{tabular}{|c|c|c|c|c|}
\hline 1 & 2 & 3 & 4 & 5 \\
\hline 6 & 7 & 8 & 9 & 10 \\
\hline 11 & 12 & 13 & 14 & 15 \\
\hline 16 & 17 & 18 & 19 & 20 \\
\hline 21 & 22 & 23 & 24 & 25 \\
\hline
\end{tabular}

\begin{tabular}{|c|c|c|c|c|}
\hline 1 & 2 & 3 & 4 & 5 \\
\hline 6 & 7 & 8 & 9 & 10 \\
\hline 11 & 12 & 13 & 14 & 15 \\
\hline 16 & 17 & 18 & 19 & 20 \\
\hline 21 & 22 & 23 & 24 & 25 \\
\hline
\end{tabular}

Tecla 18 apertada

\begin{tabular}{|c|c|c|c|c|}
\hline 1 & 2 & 3 & 4 & 5 \\
\hline 6 & 7 & 8 & 9 & 10 \\
\hline 11 & 12 & 13 & 14 & 15 \\
\hline 16 & 17 & 18 & 19 & 20 \\
\hline 21 & 22 & 23 & 24 & 25 \\
\hline \multicolumn{5}{|c}{ Tecla 19 apertada } \\
\hline
\end{tabular}

\begin{tabular}{l|l|l|l|l|}
21 & 22 & 23 & 24 & 25 \\
\hline \multicolumn{3}{|c|}{ Tecla 14 apertada }
\end{tabular}

\begin{tabular}{|c|c|c|c|c|}
\hline 1 & 2 & 3 & 4 & 5 \\
\hline 6 & 7 & 8 & 9 & 10 \\
\hline 11 & 12 & 13 & 14 & 15 \\
\hline 16 & 17 & 18 & 19 & 20 \\
\hline 21 & 22 & 23 & 24 & 25 \\
\hline
\end{tabular}

Tecla 21 apertada

\begin{tabular}{|c|c|c|c|c|}
\hline 1 & 2 & 3 & 4 & 5 \\
\hline 6 & 7 & 8 & 9 & 10 \\
\hline 11 & 12 & 13 & 14 & 15 \\
\hline 16 & 17 & 18 & 19 & 20 \\
\hline 21 & 22 & 23 & 24 & 25 \\
\hline
\end{tabular}

Tecla 15 apertada 


\begin{tabular}{|c|c|c|c|c|}
\hline 1 & 2 & 3 & 4 & 5 \\
\hline 6 & 7 & 8 & 9 & 10 \\
\hline 11 & 12 & 13 & 14 & 15 \\
\hline 16 & 17 & 18 & 19 & 20 \\
\hline 21 & 22 & 23 & 24 & 25 \\
\hline \multicolumn{5}{|c|}{ ecla 22 apertada }
\end{tabular}

\begin{tabular}{|c|c|c|c|c|}
\hline 1 & 2 & 3 & 4 & 5 \\
\hline 6 & 7 & 8 & 9 & 10 \\
\hline 11 & 12 & 13 & 14 & 15 \\
\hline 16 & 17 & 18 & 19 & 20 \\
\hline 21 & 22 & 23 & 24 & 25 \\
\hline
\end{tabular}

Tecla 23 apertada

De fato, conseguimos apagar todas as luzes, lembrando que podemos apertas as teclas em qualquer ordem que mesmo assim iremos apagar todas as luzes.

\section{DISCUSSÃO}

Este trabalho apresenta o jogo Lights out e seu problema em termos que apagar todas as luzes de forma que apertamos um número menor possível de teclas. Diante disso, através do estudo deste jogo, e toda a sua parte teórica, encontramos uma maneira em que temos um resultado rápido e prático de acharmos a solução do problema, assim seguindo a sequência de teclas dada, conseguimos chegar ao nosso objetivo. Neste caso, não foi trabalhado superficialmente a parte teórica do jogo, buscamos entender todo o conceito básico de matriz, até chegarmos ao conteúdo necessário para trabalharmos o problema.

\section{CONCLUSÃO}

Para estudantes do ensino médio é de extrema importância que o conceito matemático seja construído junto com ele raciocinando juntos, lembrando conceitos anteriores e descobrindo novos, para que assim ele consiga fazer conexões entre os assuntos e com certeza absorve muito mais conhecimento e prática matemática. E essa prática será bem mais prazerosa e satisfatória se esses estudantes utilizarem tecnologia para chegar ao resultado desejado, utilizamos um software tão quase poderoso quanto MATLAB, MATHEMATICA, entre outros do gênero numérico, mas com a seguinte vantagem, de ser um software livre, então temos uma excelente ferramenta de auxílio no ensino de matrizes, geometria analítica, gráficos em geral. Assim como existem outras ferramentas tecnológicas para auxiliar no desenvolvimento de outros conteúdos.

\section{BIBLIOGRAFIA CONSULTADA}

COELHO, F.; LOURENÇO, M.L. Um curso de álgebra linear. 2. ed. São Paulo: Editora da Universidade de São Paulo, 2010.

GONÇALVES, M.I.C. Apagando as luzes usando álgebra linear. Universidade Federal de Santa Catarina, ano.

GADELHA FILHO, D. Apostila do Scilab. Disponível em:

http://euler.mat.ufrgs.br/giacomo/Manuaissoftw $\angle S C I L A B / A p o s t i l a \% 20$ de\%20Scilab\%20\%20atualizada.pdf. Acesso em: 11 out. 2014.

RUGGIERO, M.A.G.; LOPES, V.L.R. Cálculo numérico: aspectos teóricos e computacionais. São Paulo: McGraw-Hill, 1998. 